\title{
A Case Study on Service and Management of the Emperor Qinshihuang's Mausoleum Site Museum
}

\author{
Yongzhen Zhou \\ School of Law and Business, Chifeng University, Chifeng, China \\ Email:275036285@qq.com
}

How to cite this paper: Zhou, Y. Z. (2021) A Case Study on Service and Management of the Emperor Qinshihuang's Mausoleum Site Museum. Journal of Service Science and Management, 14, 576-584. https://doi.org/10.4236/jssm.2021.146036

Received: November 26, 2021

Accepted: December 17, 2021

Published: December 20, 2021

Copyright $\odot 2021$ by author(s) and Scientific Research Publishing Inc. This work is licensed under the Creative Commons Attribution International License (CC BY 4.0).

http://creativecommons.org/licenses/by/4.0/

\begin{abstract}
The Emperor Qinshihuang's Mausoleum Site Museum (Terra-cotta Warriors Museum) was assessed as one of the first batch of $5 \mathrm{~A}$ tourist attractions in China in 2007. Taking the Emperor Qinshihuang's Mausoleum Site Museum as an example, this case analyzes the current situation of product development and quality management, and points out that improving the service quality of tourist attractions can greatly enhance the attraction of tourist attractions. This case provides reference for the development of tourist attractions. It can be said that cultural creativity is an important factor for the vitality of the Emperor Qinshihuang's Mausoleum Site Museum.
\end{abstract}

\section{Keywords}

Museum, A-Level Tourist Attractions, Historical Tourism, Cultural and Creative Product Development

\section{Introduction}

The Emperor Qinshihuang's Mausoleum Site Museum is located in Lintong district, Xi'an city, Shaanxi province. It is a large-scale Ruins Museum Based on the Museum of terracotta warriors and horses, and he Qinshihuang Mausoleum (Lishan Garden) as the backing. The establishment of Qinshihuang Mausoleum Museum relies on the archaeological discovery of terracotta warriors and horses. It has developed from the Museum of terracotta warriors and horses to the Emperor Qinshihuang's Mausoleum Site Museum, and has become a research center (Li, 2020). The Emperor Qinshihuang's Mausoleum Site Museum (museum of terracotta warriors and horses) was rated as one of the first batch of $5 \mathrm{~A}$ tourist attractions in China in 2007. Besides, it is a national first-class museum and na- 
tional archaeological site park in China. It has been ranked in the Top 25 of the "World's Most Popular Museums" by TripAdvisor, a leading global travel platform, from 2014 to 2018.

Located in Lintong district, Xi'an city, Shaanxi province, the Emperor Qinshihuang's Mausoleum Site Museum (Terra-cotta Warriors Museum) is one that is specialized in relic display. The main object exhibited here is the terra-cotta figures of more than 2000 years old. These figures stand for the garrison force that protects Emperor Qinshihuang's mausoleum. March 4th 1961, the China State Council declared Emperor Qinshihuang's mausoleum a major historical and culture sites protected at the national level, one of the first sited in the country. The mausoleum including the pits of Terra-cotta Warriors and Horses was listed as one of the UNESCO World Cultural Heritage Site on December 7th 1987 (Liu, 2019). The emperor qinshihuang's mausoleum site museum is a national first-class museum and a national archaeological site park in China.

\section{The Number of Tourists Has Repeatedly Hit Record Highs}

The Terra-cotta Warriors were discovered in 1974. In 1979, Terra-cotta Warriors Museum was completed and opened (now known as the Emperor Qinshihuang's Mausoleum Site Museum). As of September 2019, the Emperor Qinshihuang's Mausoleum Site Museum has been open to tourists for 40 years and has received more than 120 million tourists from home and abroad, including 224 heads of state and government (data from the official website of the Emperor Qinshihuang's Mausoleum Site Museum, http://www.bmy.com.cn/). The Emperor Qinshihuang's Mausoleum Site Museum is one of the earliest tourist attractions that implemented differentiated tickets and online ticket booking in Table 1 and Table 2.

In recent years, the total number of visitors and ticket revenue of the Emperor Qinshihuang's Mausoleum Site Museum have been growing continuously, and the growth rate is fast and large. In 2018, the Emperor Qinshihuang's Mausoleum Site Museum received a total of 8.58 million visitors, an increase of $25.8 \%$ compared with the same period in 2017 (Figure 1). The ticket revenue exceeded 1 billion yuan for the first time, an increase of $12.88 \%$ compared with the same period in 2017. Both the number of visitors and ticket revenue reached a new

Table 1. Ticket information of the emperor Qinshihuang's mausoleum site museum.

\begin{tabular}{cl}
\hline Ticket Price & $\begin{array}{l}120 \text { yuan/person (with only one ticket, visitors can visit the whole } \\
\text { museum) }\end{array}$ \\
March 1th - November 30th 8:30 - 17:00 \\
December 1th - February 28th 8:30 - 16:30 \\
Tips & 1) Tickets must be kept clean. After admission, please continue to \\
save the tickets for the later check. \\
2) The ticket is valid on the day, all will not be refunded.
\end{tabular}

Source: according to the official data of the Emperor Qinshihuang's Mausoleum Site Museum. 
Table 2. Online booking process of Qinshihuang's mausoleum museum.

1 The ticket price of the museum is 120 yuan per person time.

The online booking of Qinshihuang's Mausoleum Museum can be purchased

7 days in advance, and the ticket office (manual ticket window and self-service

equipment) can sell tickets. Ticket selling time: 8:30 - 17:00 from March 16 to

November 15; 8:30 - 16:30 from November 16 to March 15.

3

The ticket checking time of Qinshihuang's Mausoleum Museum is:

March 16 - November 15, 8:30 - 18:00; November 16 - March 15, 8:30 - 17:30.

Full time undergraduate students and below can enjoy half price discount with valid Student ID card (adult colleges, on-the-job education, online education, etc. do not enjoy discount). Please bring valid Student ID card and ID card to visit the park.

Visitors can take a free shuttle bus to visit the Museum of terracotta warriors

5 and horses and the beautiful mountain garden of the mausoleum of the first emperor of Qin Dynasty.

If you are successful in booking, please bring a valid second-generation ID

6 card and brush it at the ticket gate. After admission, please keep the valid ID card properly for inspection.

7 Tickets are valid on the same day. Please arrange your time to visit the park.

Tourists who do not use the ticket within the specified time and choose the wrong visit date or ticket type can apply for refund within 30 days if they do not use the ticket. After the system audit, the ticket money will be returned within 15 - 20 working days.

9 Admission to the hospital should be based on the same person and certificate.

Source: according to the official data of the emperor Qinshihuang's mausoleum site museum.

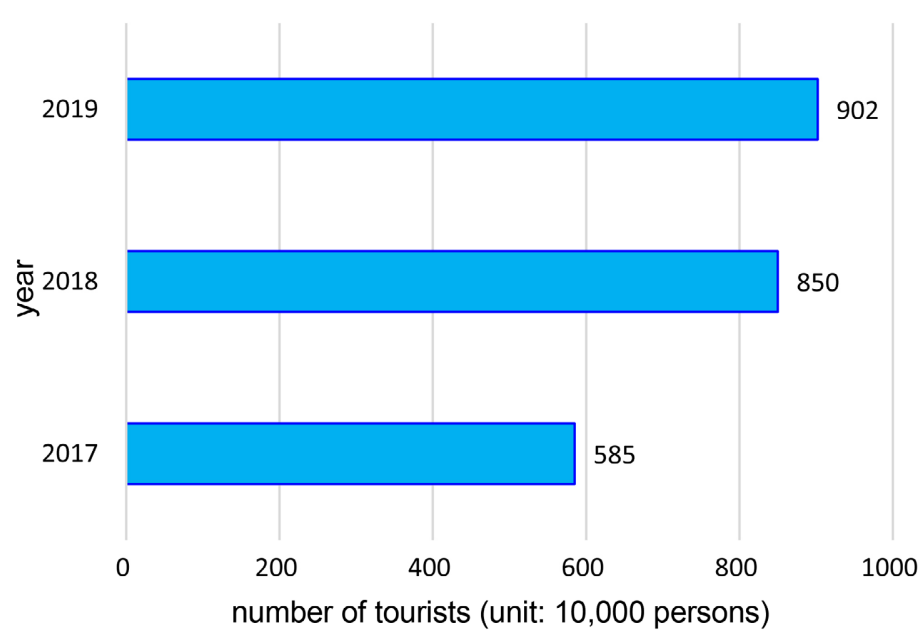

Figure 1. Number of tourists in 2017 - 2019 (unit: 10,000 persons). Source: according to the official data of the Emperor Qinshihuang's Mausoleum Site Museum.

record, second in the country after the Palace Museum in terms of the number of visitors received. 


\section{Service and Management Characteristics}

\subsection{Played a Significant Social Education Function}

The Emperor Qinshihuang's Mausoleum Site Museum gives full play to the missionary function of the museum, and has played a significant social education function since its establishment in 1979. Since the 1990s, the Emperor Qinshihuang's Mausoleum Site Museum has made reservations free visits to groups. According to the statistics of the Social Education Department of the Qinshihuang Mausoleum Museum, over the past 40 years since its establishment, a total of 3850 groups of 188,767 people have been received for free visits by university, middle school and elementary school students, more than 770,000 minors have been received. It has planned and successfully held more than 1000 activities, reaching tens of millions of people. On this basis, the museum has actively extended social education activities from inside to outside, entering schools and communities in Xi'an and Shaanxi, actively carrying out cultural relics explanations, special lectures, knowledge contests, interactive experiences and other types of activities, focusing on the educational objects participation and experience, highlighting entertaining and entertaining. While carrying out traditional publicity and education activities, it actively tries to explore new forms and methods of museum publicity and education. After years of accumulation, the museum has a total of 33 sub-projects in 12 categories of mature education activities (Table 3), and its long-term opening and operation has a very good social impact.

The Emperor Qinshihuang's Mausoleum Site Museum continues to promote the concept of "museum + Internet" integrated development, which effectively expanded the museum's online publicity and influence. While continuing to strengthen the cultural radiation of the Emperor Qinshihuang's Mausoleum Site Museum, it correctly guided the public to enter the museum to experience the

Table 3. The museum of the emperor Qinshihuang's mausoleum site museum in 2016.

\begin{tabular}{cc}
\hline Time & January - March \\
Every Tuesday, Friday \\
$9: 30$ - 11:30, 14:30 - 16:30 \\
Learn to paint terracotta warriors \\
Learn to repair terracotta warriors \\
Learn to trim Qin's hairstyle \\
Learn to write Qin's characters \\
Learn traditional paper cutting \\
Location \\
Participants \\
Mays of Registration \\
Primary and middle school students visiting the \\
Emperor Qinshihuang's Mausoleum Site Museum \\
\end{tabular}

Source: according to the official information of the Emperor Qinshihuang's mausoleum site museum. 
traditional Chinese culture (Li, 2013). At the same time, the Emperor Qinshihuang's Mausoleum Site Museum actively launched online education activities to allow visitors to learn about the Qin warriors and Qin culture in a variety of ways, and timely through the official WeChat, the Ministry of Social Education WeChat, the official WeChat, which achieved good social benefits.

\subsection{Improve Smart Management and Service Levels}

The Emperor Qinshihuang's Mausoleum Site Museum continuously strengthens cooperation with professional institutions, continuously excavates existing cultural and museum resources through professional technical channels, actively combines current technological development trends, introduces Internet thinking and technology, and develops a series of smart tourism products. In recent years, the Emperor Qinshihuang's Mausoleum Site Museum has established strategic partnerships with Baidu and Tencent, and has continuously strengthened the cultural promotion of the combination of the museum's unique cultural heritage and the Internet, effectively expanding the scope of cultural heritage dissemination.

The first is to cooperate with Baidu to restore and interpret the scenes of terracotta army pits, including interactive voice explanations on the resurrection of the warriors and war scenes, etc., using artificial intelligence technology, combined with historical relics and materials to develop, design, package and spread the culture of the Terra cotta.

The second is to explore the highlights of the museum and create brand-new cultural symbols through cooperation with Tencent, using Tencent's mature products-WeChat public account, scene-based services, audio albums, smart mobile parks, mini games, and WeChat levels (Wang, 2019).

\subsection{Focus on Improving Infrastructure}

First, the visitor center is fully functional. The tourist center of the Emperor Qinshihuang's Mausoleum Site Museum is in line with industry standards. The appearance and shape of tourists do not damage the existing landscape. It can provide tourists with information, consultation, tour arrangements, explanations, education, rest and other tourist facilities and service functions. The tourist center is reasonably located (Figure 2), with moderate scale and complete service facilities and functions.

Second, the identification system is complete. Various guide signs (including guide maps, sign boards, etc.) have outstanding shapes and characteristics, with a certain sense of art and culture, various sign systems are commensurate with the overall environment, signs and scenery the introduction card is set reasonably. Road direction signs, such as road signs, public toilet signs, and parking lot signs are accurate. Various signs have been systematically designed and produced in combination with their own cultural characteristics in terms of graphics, appearance, and materials, and relevant symbols and patterns of the signage system are clear. 


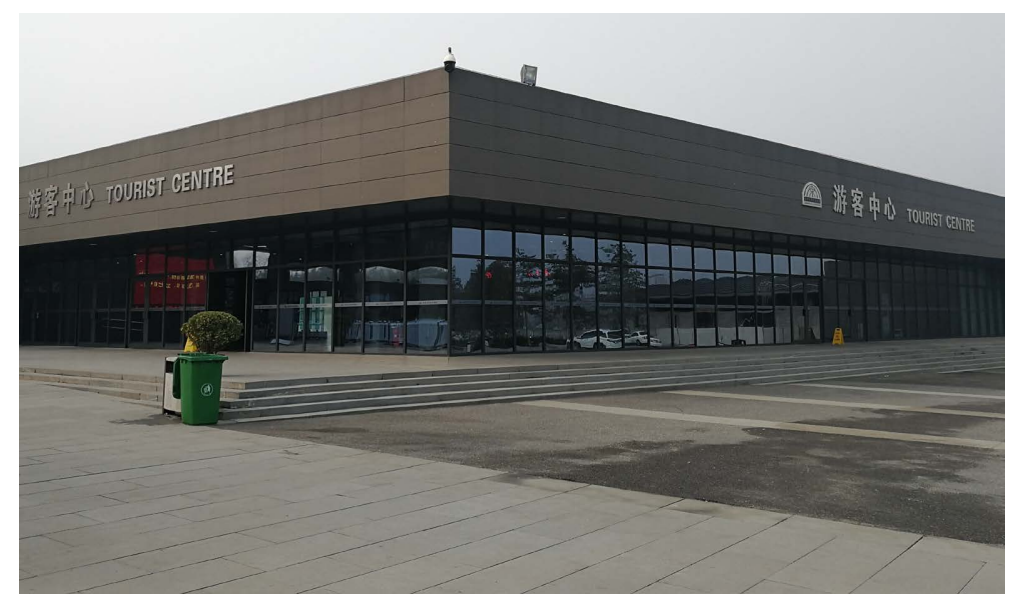

Figure 2. Tourist center of the emperor Qinshihuang's mausoleum site museum. Source: from the author.

Third, do a good job of safety protection and special group service. The third is to conscientiously do well in safety protection work and special population services. The Qinshihuang Mausoleum Museum has done a good job in the safety prevention of tourist attractions, ensured that the safety facilities for tourists to visit are complete, and continuously strengthened the construction of safety protection facilities in dangerous areas, including safety barriers, safety warning signs and other facilities and equipment, which are complete and effective. Facilities such as ticketing channels, guide signs, safety warning signs, tour trails, rest pavilions, environmentally friendly toilets and safety fences can be routinely inspected and maintained in an orderly manner, and all kinds of barrier free facilities and other special crowd channels can always be kept unblocked.

\subsection{Pay Attention to the Characteristic Innovation and Development of Tourism Products}

The Emperor Qinshihuang's Mausoleum Site Museum has been actively developing cultural and creative products since 2017, which not only enriches the supply of tourism products, but also promotes the industrialization, specialization and aggregation of tourism commodities, and actively develops its own cultural and creative products.

At the same time, the Emperor Qinshihuang's Mausoleum Site Museum also held a cultural and creative product development competition to continuously explore creative products with a strong sense of design and high quality (Peng, 2019). Self-service vending machines have also been set up in the scenic area to enhance the convenience of selling tourist products, which are generally welcomed by tourists and effectively promote the innovative development of characteristic tourist products.

In recent years, the Emperor Qinshihuang's Mausoleum Site Museum has also increased the development of cultural derivative products. In addition to traditional souvenirs, such as postcards, commemorative coins, commemorative med- 
als, key chains, porcelain cups, and T-shirts with Qin terra cotta warriors and horses, it also actively highlights various characteristic publications, such as the multi-language version of "World the Eighth Wonder of the Qin Terracotta Warriors", "The Treasures of the Qin Mausoleum, Bronze Chariots and Horses" and "Fantasy Legion" and other large-scale books, albums and CDs.

In product development, we have continuously strengthened the protective use of cultural symbols, strengthened the protective publicity of famous brands and cultural symbols such as "Qin terra cotta Museum", "Bronze chariots and horses", "Qin Terracotta cotta warriors and horses", "General Warriors", and improved the product radiation scope and marketing power of the Emperor Qinshihuang's Mausoleum Site Museum.

\subsection{Build an Official Website and Official WeChat}

One is to create a fully functional official website. The Emperor Qinshihuang's Mausoleum Site Museum uses modern network technologies, such as graphical interfaces, multimedia, etc. (Huang \& Chen, 2020), to display the tourism products and service content of the tourist attractions on the official website, so that potential customers can get to know the Emperor Qinshihuang's Mausoleum Site Museum at home, and can clear related service items. The official website of the Emperor Qinshihuang's Mausoleum Site Museum (website:

http://www.bmy.com.cn/, Figure 3), details the development history of Qin Shi Huang Mausoleum Museum, the columns set up generally have the effect of popular science, and the whole website has the basic functions of multimedia display, cultural and creative product sales, ticket pre-sale and so on.

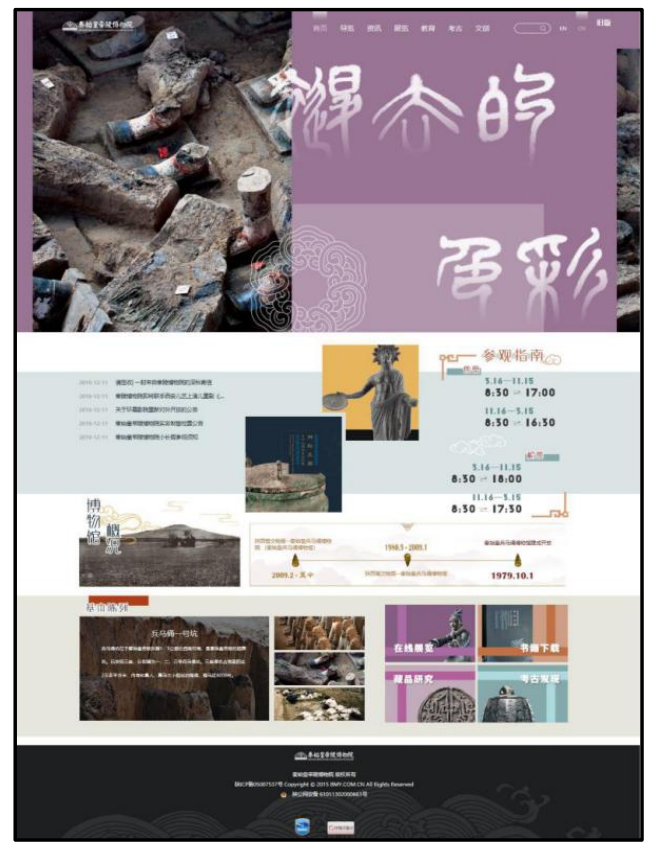

Figure 3. Official website of the emperor Qinshihuang's mausoleum site museum. Source: from of the official website information of the Emperor Qinshihuang's Mausoleum Site Museum. 

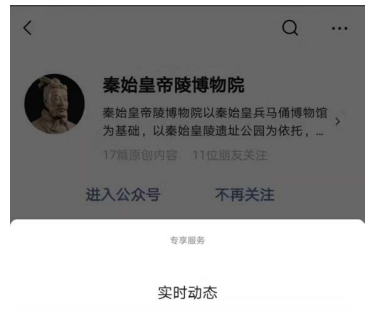

微信购票

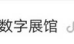

智慧导览

语音画冊

取消
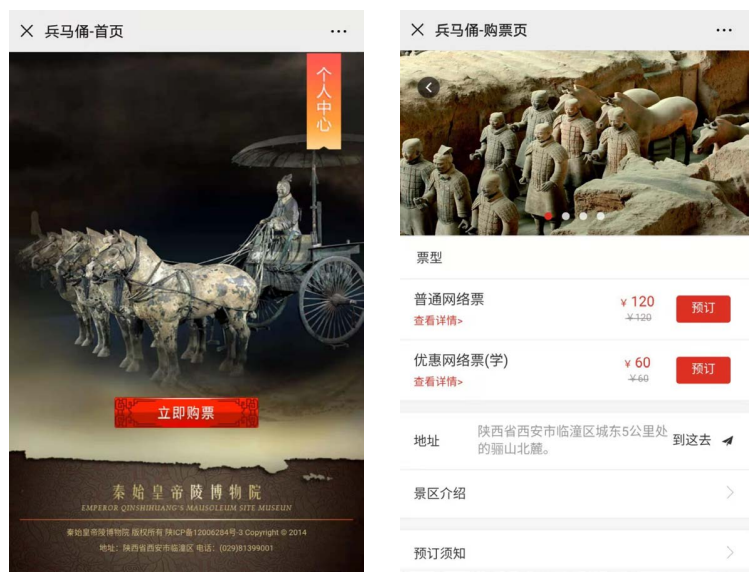

Figure 4. Official WeChat of the emperor Qinshihuang's mausoleum site museum. Source: from of the official WeChat information of the Emperor Qinshihuang's mausoleum site museum.

The second is to give full play to the participatory function of official WeChat. The official WeChat of the Emperor Qinshihuang's Mausoleum Site Museum has not only navigation, tourist guides, shopping guides and other services, but also travel guides, route recommendations, catering, accommodation, shopping and other information, which effectively ensures convenient and fast inquiries for tourists. At the same time, small columns such as "real-time dynamic" can dynamically query or inquire about the maximum carrying capacity of tourists in the scenic spot and the expected number of tourists in a specific period of time, so as to facilitate tourists to choose travel time. The official WeChat has established and improved the e-commerce system, and tourists can effectively book tickets, accommodation, catering, goods or other personalized services through the official WeChat channel (Figure 4).

\section{Conclusion}

As the core element of attracting tourists to travel, tourist attractions play a vital role in promoting the development of the tourism industry. At present, many tourist attractions have not effectively managed the actual needs of tourists and the utilization of resources, resulting in tourists not having a better travel experience in the process of playing. Therefore, the concept of experience should be integrated into the development of the tourist attractions, and a perfect management mode should be constructed in combination with the current situation of market development and the specific needs of tourists so as to effectively improve the effectiveness of scenic spot management and promote the better development of tourism.

The development of cultural and creative products of the Emperor Qinshihuang's Mausoleum Site Museum pays attention to "let cultural relics live", and carries out creative transformation on the basis of in-depth excavation and interpretation of traditional culture. Cultural and creative products have become the perfect footnote of traditional culture. With the advent of the big data era 
brought by the Internet, Internet plus has become a new form of museum culture industry. The Emperor Qinshihuang's Mausoleum Site Museum continuously promotes cultural and creative renovation, and the creation of products is promoted by Internet plus, expanding the methods of communication and sales channels. With the help of the Internet, the cultural and creative products of the Emperor Qinshihuang's Mausoleum Site Museum have continuously increased their popularity and effectively promoted consumers' cognition of traditional culture.

\section{Acknowledgements}

This case was compiled with reference to the official website of the Emperor Qinshihuang's Mausoleum Site Museum (http://www.bmy.com.cn/), official WeChat (WeChat ID: bmy1979), and the website of China National Cultural Heritage Administration (http://www.sach.gov.cn).

\section{Funding}

This work is supported by the Chifeng University Tourism and Cultural Industry Innovation Research Team Program (Cfxykycxtd202003), the Chifeng University First Class Discipline Construction Program (2020305).

\section{Conflicts of Interest}

The author declares no conflicts of interest regarding the publication of this paper.

\section{References}

Huang, W., \& Chen, C. J. (2020). A Contrastive Analysis of Perceived Image and Projected Image-Taking Emperor Qinshihuang's Mausoleum Site Museum as an Example. Journal of Sichuan Tourism University, 33, 63-69.

Li, B. (2020). Integration of Site Display and Cultural Relic Protection of Heritage Museum: A Case Study of Qinshihuang's Mausoleum Site Museum. China Museum, 37, 77-82.

Li, X. J. (2013). Cultural Tourism, Yourism Gaze and Others. Tourism Tribune, 28, 8-9.

Liu, L. (2019). An Overview of Qinshihuang's Mausoleum Site Museum. Identification and Appreciation of Cultural Relics, 10, 80-82.

Peng, J. (2019). Research on the Creation of Qinshihuang's Mausoleum Site Museum in the Internet Plus Background. Beauty and the Times, 18, 94-97.

Wang, J. H. (2019). Research on Operation of the Wechat Platform of "Emperor Qinshihuang'S Mausoleum Site Museum”. Northwest University of Technology, 10, 11-25. 